\title{
Perfil etnobotânico de plantas medicinais comercializadas em feiras livres de São Luís, Maranhão, Brasil
}

\author{
Ethnobotanical profile of medicinal plants sold in fairs of São Luís, Maranhão, Brazil
}

\author{
M.M.C.Cunha ${ }^{1}$; R.S.D.Gondim ${ }^{1}$; B.F.Bonfim ${ }^{1}$; N.J.P. Batalha Junior ${ }^{1}$; \\ W.A.Barroso ${ }^{1,2^{*}} ;$ C.M. Vilanova ${ }^{1}$ \\ ${ }^{I}$ Departamento de Farmácia, Laboratório de Farmacobotânica, Universidade Federal do Maranhão, CEP: \\ 65080-805, São Luís-Maranhão, Brasil. \\ ${ }^{2}$ Departamento de Clínica Médica, Laboratório de Investigação Médica 51 (LIM-51), Universidade de São \\ Paulo, CEP: 05403-000, São Paulo-São Paulo, Brasil. \\ *wermersonbarroso@usp.br
}

(Recebido em 14 de setembro de 2015; aceito em 25 de outubro de 2015)

Foi realizado um levantamento de plantas medicinais comercializadas em feiras livres do município de São Luís, Maranhão, Brasil, em 2013. Utilizaram-se questionários semiestruturados que abordaram o nome popular da planta, bem como a parte utilizada e forma de preparo, além da finalidade terapêutica.

Os resultados mostraram 41 espécies na categoria medicinal distribuídas em 27 famílias, sendo Lamiaceae (18\%) e Asteraceae (7\%) com maior número de espécies. A planta mais citada foi Ocimum basilicum L. (16\%), conhecida popularmente por manjericão. A decoç̧ão foi o método prevalente de preparo $(58 \%)$ e a folha foi o órgão mais utilizado nas preparações $(73 \%)$. Em relação à finalidade terapêutica, observou-se maior utilização em casos de doenças que envolvem problemas infecciosos, inflamatórios e respiratórios, sendo os homens os principais detentores do conhecimento sobre as plantas medicinais. Observou-se que a maioria das indicações relatadas pelos feirantes foi similar às encontradas na literatura científica pesquisada.

Palavras-chave: Etnobotânica, São Luís, Plantas medicinais.

A survey of medicinal plants marketed in free fairs was performed in the city of São Luís, Maranhão, Brazil, in 2013. Was used a semi-structured questionnaire that addresses the common name of the plant, as well as the portion used and the way of preparation, besides of the therapeutic purposes. The results showed 41 species in the medical category distributed in 27 families, being Lamiaceae (18\%) and Asteraceae (7\%) with the highest number of species. The most cited plant was Ocimum basilicum L. (16\%), popularly known as basil. The decoction was the prevalent method of preparation (58\%) and the leaf was the organ most commonly used in preparations (73\%). With regard to therapeutic purpose, there was greater use in cases of diseases involving infectious problems, inflammatory and respiratory, being the men the main holders of knowledge about medicinal plants. It was observed that most of the indications reported by merchants were found in any type of scientific literature.

Keywords: Ethnobotany, São Luís, Medicinal plants. 


\section{INTRODUÇÃO}

Os produtos naturais são utilizados desde os primórdios da humanidade. A busca por alívio e cura de doenças pela ingestão de ervas e folhas talvez tenham sido uma das primeiras formas de sua utilização [1]. As práticas relacionadas ao uso popular de plantas medicinais são transmitidas oralmente de geração em geração e os estudos etnofarmacológicos são de suma importância para a preservação deste conhecimento [2].

A etnobotânica é a ciência que analisa e estuda as informações populares que o homem tem sobre o uso das plantas. É através dela que se mostra o perfil de uma comunidade e seus usos em relação às plantas, pois cada comunidade tem seus costumes e peculiaridades, visando extrair informações que possam ser benéficas sobre usos de plantas medicinais [3].

$\mathrm{O}$ uso de plantas medicinais pela população mundial tem sido muito significativo nos últimos tempos. Dados da Organização Mundial de Saúde (OMS) mostram que cerca de $80 \%$ da população mundial fez uso de algum tipo de planta na busca de alívio de alguma sintomatologia desagradável [4]. Ainda hoje nas regiões mais pobres do Brasil e até mesmo nas grandes cidades, plantas medicinais são comercializadas em feiras livres, mercados populares e encontradas em quintais residenciais [5].

Dentro desse contexto, o presente trabalho teve como finalidade realizar um estudo etnobotânico das plantas medicinais vendidas nas feiras livres do município de São Luís, Maranhão, Brasil, com o intuito de verificar o conhecimento popular existente na cidade sobre as plantas e a eficácia no tratamento das doenças. Tendo como tentativa evitar os malefícios decorrentes do uso indevido das plantas medicinais, por meio de orientações prestadas durante a execução da pesquisa quanto à forma correta de preparação de cada parte da planta a ser utilizada, à quantidade estimada de planta a ser usada em cada preparação e à integridade das plantas para o uso, proporcionando assim um aumento dos benefícios na sua utilização.

\section{MATERIAL E MÉTODOS}

O estudo constituiu-se de um levantamento etnobotânico sobre as plantas medicinais comercializadas nas feiras livres do município de São Luís - Maranhão, sendo os locais de coleta de informações o Mercado Central e as feiras da Cohab, Cidade Operária e Liberdade. O estudo foi de caráter quantitativo, descritivo e exploratório, mediante aplicação de questionários semiestruturados. Os questionários foram aplicados a sete feirantes que comercializavam plantas nos quatro pontos de estudo, em 2013.

Buscou-se inicialmente fazer uma caracterização do perfil dos feirantes quanto à idade, gênero, renda mensal, meios de comunicação utilizados e aquisição do conhecimento. Em seguida, um levantamento sobre o conhecimento e utilização das plantas medicinais, quanto às partes utilizadas, formas de preparo e modos de obtenção das plantas.

Os feirantes participantes da pesquisa assinaram o Termo de Consentimento Livre e Esclarecido (TCLE). Os dados foram analisados através de cálculos percentuais e de frequência relativa pelo software Microsoft Excel $^{\circledR}$ e dispostos na forma de gráficos e tabelas. A pesquisa foi aprovada pelo Comitê de Ética em Pesquisa (CEP) da Universidade Federal do Maranhão (UFMA), sob o parecer de ${ }^{\circ} 154.698$.

\section{RESULTADOS E DISCUSSÃO}

Foram analisados os questionários dos sete feirantes entrevistados, onde se observou que $43 \%$ têm idades variando entre 40 e 59 anos, sendo a maioria constituída por homens (57\%), tendo renda salarial de até um salário mínimo, correspondendo a $42 \%$ do total, seguidos por $28 \%$ que possuem renda de um a três salários mínimos e utilizam como meio de comunicação a $\mathrm{TV}$, rádio e celular na mesma frequência, com um percentual de $29 \%$. Seguida da internet com 13\% (Tabela 1).

Em estudo realizado por Fuck et al. (2005) [6], foi observado que pessoas encontradas na faixa etária superior a 30 anos têm uma menor resistência a participar de pesquisas e se dispõem 
a conversar e fornecer informações sobre plantas medicinais. Segundo Calábria (2008) [7] os homens detém maior conhecimento das plantas nativas, enquanto as mulheres possuem melhores conhecimentos de plantas cultivadas que crescem próxima a casa, no quintal e no roçado.

Arnous et al. (2005) [8], em um estudo feito em Datas-MG, comprovou que os principais meios de comunicação utilizados por seus entrevistados foram o rádio e a televisão, o que comprova serem ainda os meios de comunicação mais acessíveis a população de modo geral.

Quanto às formas de aquisição de conhecimento sobre plantas medicinais (Figura 1A) observou-se que o conhecimento adquirido pelos feirantes foi oriundo principalmente das pessoas mais experientes, que já trabalham nas feiras há mais de 20 anos $(62 \%)$, dos amigos e vizinhos (25\%) e por conta própria (13\%). As observações refletem a importância da transmissão oral das práticas terapêuticas tradicionais, bem como o caráter familiar da comercialização dos recursos em questão [9].

Em relação às formas de obtenção das plantas medicinais, observou-se que a maioria dos feirantes adquiriu plantas de terceiros (100\%). Resultados semelhantes foram encontrados na pesquisa de Rocha et al. (2012) [9], em estudo feito na feira livre do município de Lagoa Nova$\mathrm{RN}$, onde todos os feirantes adquiriam as plantas que comercializavam a partir de terceiros.

Tabela 1: Distribuição numérica e percentual das variáveis socioeconômica e demográfica dos sete entrevistados nas feiras da Cohab, Cidade Operária, Liberdade e Mercado Central. Município de São Luís, Estado do Maranhão, Brasil, 2013.

\begin{tabular}{lll}
\hline Variáveis & N & \% \\
\hline Sexo & & \\
Masculino & 4 & 57 \\
Feminino & 3 & 43 \\
Total & 7 & 100 \\
Faixa etária & & \\
$18-39$ anos & 2 & 29 \\
40-59 anos & 3 & 43 \\
$\geq 60$ anos & 1 & 14 \\
Não informou & 1 & 14 \\
Total & 7 & 100 \\
Renda salarial & & \\
Até 1 salário & 3 & 42 \\
1 a 3 salários & 2 & 29 \\
Não informado & 2 & 29 \\
Total & 7 & 100 \\
Meio de comunicação & & \\
TV & 2 & 29 \\
Celular & 2 & 29 \\
Internet & 1 & 13 \\
Rádio & 2 & 29 \\
Total & 7 & 100 \\
\hline
\end{tabular}

$N=$ Número $\%=$ Percentagem

Levando em conta as partes vegetais utilizadas para a comercialização pelos feirantes (Figura 1B), as folhas são as mais comercializadas (64\%), seguidas das cascas e raízes (14\% cada), e das flores e frutos (4\% cada). A grande utilização das folhas para a comercialização pode ser explicado pela facilidade da coleta, já que elas ficam expostas e estão presentes em grande parte da vida da planta, além de concentrarem grande parte dos princípios ativos na grande maioria das espécies [10]. Coelho et al. (2002) [11], estudando as plantas usadas por comunidades da Serra do Brigadeiro em Minas Gerais, também verificaram alta frequência no uso de folhas, tanto que nessa região as plantas medicinais são conhecidas como "folhas de 
chá". Entretanto, é importante salientar que o predomínio no uso da folha ou da casca das plantas muitas vezes está relacionado ao ambiente de ocorrência, sendo que as disponibilidades das partes das plantas variam ao longo do ano e definem a preferência pelas comunidades.

Foram identificadas variadas formas de preparo das plantas (Figura 1C), sendo as mais utilizadas a decocção (58\% dos casos) e decocção e maceração (18\% dos casos), semelhante ao encontrado por Pinto et al. (2006) [12] em comunidades rurais de Itacaré/BA.

A

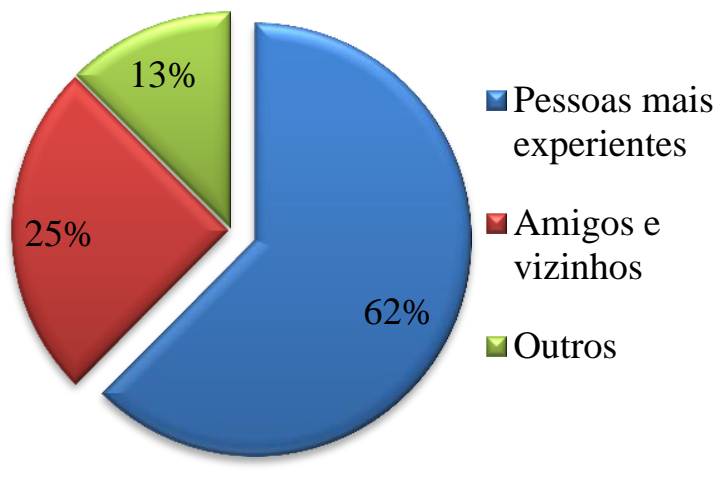

B

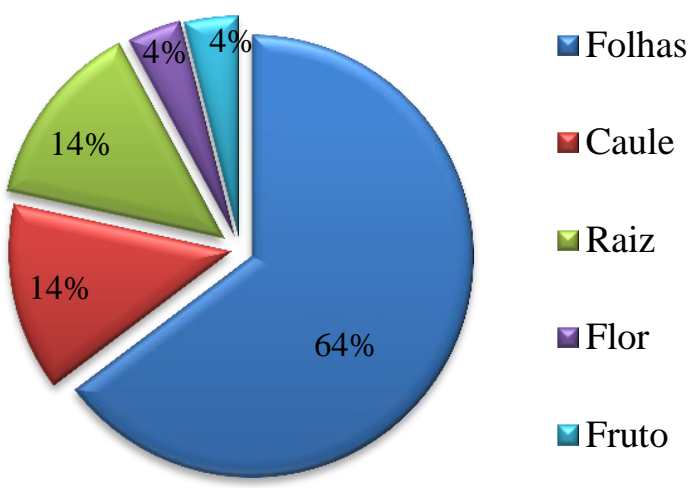

C

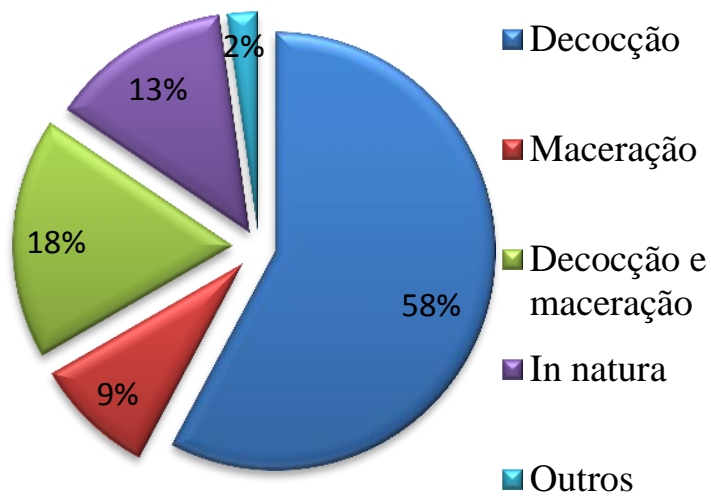

Figura 1 - Distribuição percentual da forma de aquisição do conhecimento das plantas medicinais (A), partes vegetais usadas $(B)$ e modo de preparo das plantas para utilização $(C)$ pelos sete entrevistados nas feiras da Cohab, Cidade Operária, Liberdade e Mercado Central. Município de São Luís, Estado do Maranhão, Brasil, 2013. 
Quanto a quem recorrem primeiramente em caso de doenças (Figura 2A), 71\% dos entrevistados recorrem a plantas medicinais e $29 \%$ aos médicos. Segundo Silveira et al. (2008) [13], a dificuldade de acesso da população à assistência médica e farmacêutica, o custo elevado dos medicamentos industrializados e uma tendência dos consumidores a utilizarem produtos de origem natural, por não os considerarem perigosos, contribuem para a prioridade na escolha do tratamento de enfermidades com plantas medicinais.

Quanto à via de administração das plantas medicinais (Figura 2B), a maioria (56\%) relatou que faz o uso por via oral. A forma tópica empregada por $27 \%$ dos entrevistados foi a segunda mais citada. Uma parcela pequena dos feirantes, $2 \%$ apenas, mencionou a administração das plantas por via nasal, através da inalação.

Os dados estão condizentes com o trabalho de Jesus et al. (2009) [14] feito na comunidade de Pirizal, na cidade de Nossa Senhora do Livramento - MT, onde a via de administração mais empregada foi a oral (100\%), sendo usadas as formas de infusão (75\%) ou macerada em água fria $(25 \%)$. No tratamento das inflamações foram preferencialmente utilizados banhos tópicos (60\%), especialmente nas inflamações do trato geniturinário.

Foram levantadas 41 espécies na categoria medicinal distribuídas em 27 famílias (Tabela 2), sendo Lamiaceae (18\%) e Asteraceae (7\%) as famílias com maior número de espécies. Entre as enfermidades apontadas como as mais frequentes destacam-se problemas de pressão arterial, problemas inflamatórios, problemas cardíacos, gripe, depressão e diabetes.

A espécie Ocimum basilicum L. (Lamiaceae) foi a mais citada (16\%), sendo utilizada para o tratamento de doenças respiratórias e dores de cabeça. Ocimum basilicum L, conhecida popularmente como manjericão, é uma espécie usada tradicionalmente como erva medicinal no tratamento de tosse, diarreia, constipação, verruga, febre e disfunções renais. Seu uso como condimento é muito antigo devido ao aroma que suas folhagens fornecem aos alimentos. É também uma fonte de compostos aromáticos e óleos essenciais contendo compostos biologicamente ativos com atividades inseticidas, nematicidas e antimicrobianas $[15,16,17]$.

\section{B}

A
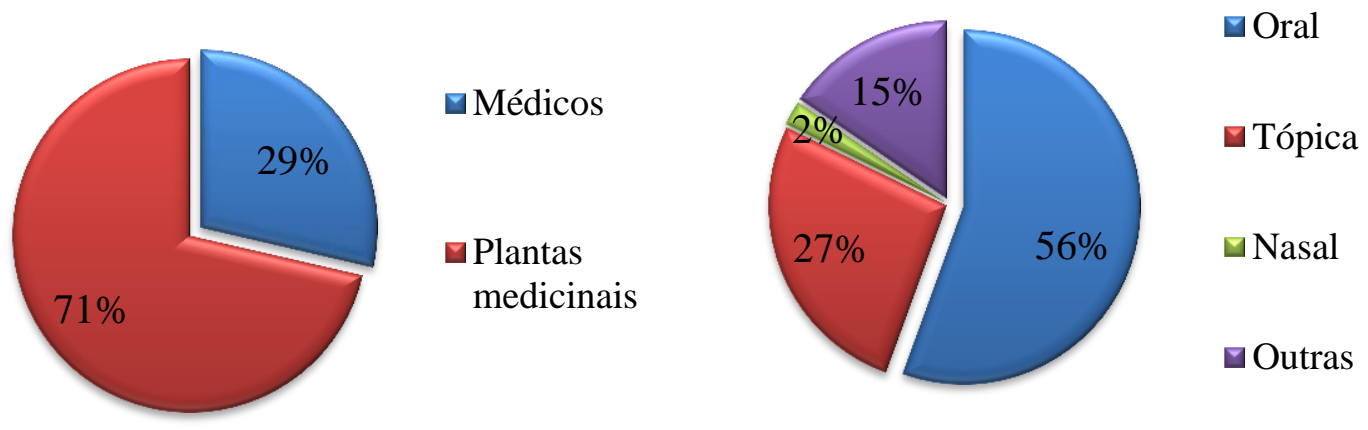

Figura 2. Distribuição percentual da prioridade na escolha do tratamento de enfermidades (A) e vias de administração das plantas medicinais $(B)$ dos sete entrevistados nas feiras da Cohab, Cidade Operária, Liberdade e Mercado Central. Município de São Luís, Estado do Maranhão, Brasil, 2013.

A espécie Chenopodium ambrosioides L. (Amaranthaceae) foi a segunda mais citada (13\%). Ela é conhecida popularmente como "erva de Santa Maria", "mastruço", "ambrosina", "mastruz", "erva-de-bicho", "mentruz" ou "erva-do-formigueiro", sendo muito empregada na medicina caseira na região centro oeste, sul e sudeste do Brasil [18]. As folhas trituradas são comumente utilizadas para alívio de dor, inflamação, como cicatrizante, em contusão, fraturas, 
problemas respiratórios, como diurética, para bronquite, tuberculose, como abortiva e em reumatismo. Entretanto, é a sua ação anti-helmíntica que a caracteriza como erva medicinal. Segundo a OMS é uma das plantas medicinais mais utilizadas entre os remédios tradicionais no mundo inteiro $[18,19,20]$.

A Ruta graveolens L. (Rutaceae), também conhecida como arruda, foi citada por $11 \%$ dos entrevistados. Ensaios farmacológicos comprovaram seu efeito como anti-helmíntica, febrífuga, abortiva, antiparasitária, espasmolítica, fotossensibilizante, cicatrizante, anti-inflamatória, antirreumática e antiulcerogênica, sudorífera [21,22]. De acordo Mejri et al. (2010) [23], a arruda também é indicada para a normalização do ciclo menstrual, repelente, vermicida, tratamento da leishmaniose, vermes como o oxiúros, combate de piolhos e outros parasitas.

Das três espécies mais citadas, duas estão presentes, Ruta graveolens L. e Chenopodium ambrosioides L., na Relação Nacional de Plantas Medicinais de Interesse ao SUS (RENISUS). Essa relação é constituída de espécies vegetais cujo critério de inclusão tem por base o potencial de avançar nas etapas da cadeia produtiva e de gerar produtos de interesse ao SUS [24]. 
Tabela 2: Informações sobre as plantas medicinais comercializadas nas feiras da Cohab, Cidade Operária, Liberdade e Mercado Central. Município de São Luís, Maranhão, Brasil, 2013

\begin{tabular}{|c|c|c|c|c|c|c|}
\hline $\begin{array}{l}\text { Nome } \\
\text { popular }\end{array}$ & Nome científico & Família & Parte utilizada & Indicação terapêutica & Preparo & $\%(\mathbf{N})$ \\
\hline Abranda & Zebrina pendula S.. & Commelinaceae & Folhas & Calmante $^{\text {ANC }}$ & Decocção & $0,97 \%(1)$ \\
\hline Açoita cavalo & Luehea divaricata Mart. & Tiliaceae & Caule & Tosses, laringites e bronquites ${ }^{25}$ & Decocção e maceração & $1,94 \%(2)$ \\
\hline Algodão & Gossypium herbaceum L. & Malvaceae & Folhas & Cicatrizante $^{\mathrm{ANC}}$ & In natura & $3,88 \%(4)$ \\
\hline Alho & Allium sativum $L$. & Liliaceae & Bulbo & Antihelmíntico $^{26}$ & Decocção & $0,97 \%(1)$ \\
\hline Andiroba & Carapa guianensis Aubl. & Meliaceae & Caule & Antimicrobiano $^{27}$ & Decocção & $0,97 \%(1)$ \\
\hline Aroeira & Myracrodruon urundeuva Allemao & Anacardiaceae & Caule & Anti-inflamatório e antimicrobiano ${ }^{28}$ & Decocção e maceração & $3,88 \%(4)$ \\
\hline Arruda & Ruta graveolens $L$ & Asteraceae & Folhas & Antimicrobiano $^{29}$ & Decocção & $4,85 \%(5)$ \\
\hline Babosa & Aloe vera (L.) Burm. & Liliaceae & Folhas & Cicatrizante, antiulcerativo ${ }^{30}$ & In natura & $1,94 \%(2)$ \\
\hline Barbatimão & Stryphnodendron barbatimam Mart. & Fabaceae & Caule & Antisséptico e antiinflamatório ${ }^{31}$ & Decocção e maceração & $1,94 \%(2)$ \\
\hline Boldo & Peumus boldus Benth. & Lamiaceae & Folhas & Dor abdominal, flatulência e hepatoprotetor ${ }^{32,33}$ & Decocção & $2,91 \%(3)$ \\
\hline Cana-da-índia & Canna indica $L$. & Cannaceae & Folhas & Calmante ${ }^{34}$ & Decocção & $2,91 \%(3)$ \\
\hline Capim limão & Cymbopogon citratus Stapf & Poaceae & Folhas & Cólicas uterinas e intestinais $^{35}$ & Decocção & $3,88 \%(4)$ \\
\hline Cebolinha & Allium schoenoprasum L. & Alliaceae & Folhas & Digestivo, antibiótico, analgésico ${ }^{36}$ & Maceração & $0,97 \%(1)$ \\
\hline Chá de caboclo & Coccoloba argentinensis & Poligonaceae & Caule & Anemia, anti-inflamatório ${ }^{37}$ & Decocção & $0,97 \%(1)$ \\
\hline Chanana & Turnera ulmifolia $L$ & Turneraceae & Folhas & Anti-Ulcerogênica $^{38}$ & Decocção & $1,94 \%(2)$ \\
\hline Cravo de defunto & Tagetes minuta L. & Compositeae & Folhas/flor & Diarreia $^{39}$ & Outros & $2,91 \%(3)$ \\
\hline Cravo-da-índia & Caryophyllus aromaticus $L$. & Myrtaceae & Folhas & Antimicrobiano $^{40}$ & Decocção & $0,97 \%(1)$ \\
\hline Enxuga & Vernonia filantus Scherb & Asteraceae & Folhas & Inflamação nos ovários ${ }^{41}$ & Decocção & $2,91 \%(3)$ \\
\hline Erva cidreira & Lippia alba (Mill.) N.E.Br & Lamiaceae & Folhas & Calmante $^{42}$ & Decocção & $2,91 \%(3)$ \\
\hline Erva santa & Aloysia gratissima $T$. & Verbenaceae & Folhas & Antimicrobiano $^{43}$ & In natura & $0,91 \%(1)$ \\
\hline Estoraque & Styrax camporum Pohl & Styracaceae & Folhas & Antimicrobiano ${ }^{44}$ & Decocção & $0,91 \%(1)$ \\
\hline Eucalipto & Eucalyptus globulus Labill & Myrtaceae & Folhas & Inflamações pulmonares ${ }^{45}$ & Decocção & $0,91 \%(1)$ \\
\hline Favacão & Ocimum gratissimum L. & Lamiaceae & Folhas & Gripe $^{46}$ & Decocção & $0,91 \%(1)$ \\
\hline Gengibre & Zingiber officinale & Zingiberaceae & Raiz & Garganta $^{47}$ & In natura & $0,91 \%(1)$ \\
\hline Hortelãzinho & Mentha $x$ villosa Huds & Lamiaceae & Folhas & Gripe, resfriado, febre ${ }^{48}$ & Decocção e maceração & $3,88 \%(4)$ \\
\hline Insulina & Cissus sicyoides $L$. & Vitaceae & Folhas & Diabetes $^{22}$ & Decocção & $4,85 \%(5)$ \\
\hline Jalapa & Mirabillis jalapa $L$. & Nyctaginaceae & Frutos & Antibacteriana e antimicrobiana ${ }^{49}$ & Maceração e decocção & $0,97 \%(1)$ \\
\hline Jambú & Spilanthy acmella & Astaraceae & Folhas & Antimicrobiano e anti-inflamatório ${ }^{50}$ & Decocção & $0,97 \%(1)$ \\
\hline Janaúba & Himatanthus drasticus Mart. & Apocynaceae & Caule & Vermicida $^{51}$ & Decocção & $0,97 \%(1)$ \\
\hline Jardineira & Alpinia zerumbet $P$. & Zingiberaceae & Folhas & Anti-hipertensiva e tranquilizante ${ }^{18}$ & Decocção e maceração & $1,97 \%(2)$ \\
\hline Jatobá & Hymenaea stigonocarpa Mart. ex Hayne. & Leguminosae & Caule & Anti-inflamatório e depurativo ${ }^{52}$ & Decocção e maceração & $0,97 \%(1)$ \\
\hline
\end{tabular}




\begin{tabular}{|c|c|c|c|c|c|c|}
\hline Manjericão & Ocimum basilicum $L$ & Lamiaceae & Folhas & Antiespamótica e sedativa $^{53}$ & Decocção e maceração & $6,80 \%(7)$ \\
\hline Manjerona & Origanum majorana & Lamiaceae & Folhas & Antimicrobiano $^{54}$ & Decocção & $0,97 \%(1)$ \\
\hline Mastruz & Chenopodium ambrosioides $L$. & Chenopodiaceae & Folhas & Cicatrizante $^{55}$ & Maceração & $5,83 \%(6)$ \\
\hline Oriza & Pogostemon cablin (Blanco) Benth. & Lamiaceae & Folhas & Antidepressiva e anti-inflamatória ${ }^{56}$ & Decocção & $3,88 \%(4)$ \\
\hline Pariri & Arrebidaea chica & Bignoniaceae & Folhas & Cólica intestinal e anti-inflamatório ${ }^{57}$ & Decocção & $1,94 \%(2)$ \\
\hline Quebra pedra & Phyllanthus niruri $L$ & Euphorbiaceae & Raiz & Diabetes $^{58}$ & Decocção & $1,94 \%(2)$ \\
\hline Quina & Quassia amara L & Simaroubaceae & Folhas & Problemas hepáticos e gastrintestinais ${ }^{49}$ & Decocção & $0,97 \%(1)$ \\
\hline Romã & Punica granatum & Punicaceae & Fruto & Inflamação na garganta e bronquite ${ }^{59}$ & In natura & $2,91 \%(3)$ \\
\hline Vinagreira roxa & Hibiscus sabdariffa & Malvaceae & Folhas & Erisipela, emagrecimento e má digestão ${ }^{60}$ & Decocção & $1,94 \%(2)$ \\
\hline \multirow[t]{2}{*}{ TOTAL } & & & & & & $100 \%$ \\
\hline & & & & & & $(103)$ \\
\hline
\end{tabular}

Legenda: \% (N): percentagem/número de citações; ANC: atividade não comprovada. 


\section{CONCLUSÃO}

A partir dos resultados obtidos neste estudo etnobotânico pode-se perceber a importância do conhecimento popular na promoção de medidas de tratamento de diversas enfermidades. Os feirantes entrevistados conhecem e utilizam plantas medicinais geralmente como primeira forma de tratamento, devido a grande confiança nesses produtos, preços acessíveis e muitas das vezes dificuldade de acesso a programas de saúde básica. Com relação à finalidade terapêutica, observou-se maior utilização em casos de doenças que envolvem problemas infecciosos, inflamatórios e respiratórios, sendo os homens os principais detentores do conhecimento sobre as plantas medicinais. A decocção foi a principal forma de utilização indicada pelos feirantes, sendo as folhas e as cascas do caule como as partes mais vendidas.

Verificou-se que o conhecimento tradicional interage com o conhecimento científico, proporcionando resultados satisfatórios nas indicações da medicina popular. As diversas formas de se utilizar plantas medicinais as tornaram uma alternativa natural para algumas doenças ou dores. Apesar da maioria das propriedades indicadas serem confirmadas por estudos científicos realizados, deve-se promover junto a estas comunidades, esclarecimento por profissionais capacitados, sobre o modo como as preparações medicinais são feitas, a fim de minimizar possíveis problemas de intoxicação, e obter resultados positivos com a utilização destas plantas medicinais.

Os resultados obtidos nesse trabalho refletem que costumes antigos de se tratar doenças com plantas medicinais continuam sendo uma prática permanente entre os entrevistados, pelo fato desse tipo de tratamento ser economicamente viável.

\section{AGRADECIMENTOS}

À Fundação de Amparo à Pesquisa e ao Desenvolvimento Científico e Tecnológico do Maranhão (FAPEMA) e ao Conselho Nacional de Desenvolvimento Científico e Tecnológico (CNPq).

\section{REFERÊNCIAS BIBLIOGRÁFICAS}

1. Viegas Jr C, Bolzani VS, Barreiro EJ. Os produtos naturais e a química medicinal moderna. Química Nova. 2006, 29: 326 - 337.

2. Falcão, DQ \& Menezes, FS. Revisão etnofarmacológica, farmacológica e química do gênero Hyptis. Rev. Bras. Farm. 2003,84: 68 - 74.

3. Martins AG, Rosário DL, Barros MN, Jardim MAG. Levantamento etnobotânico de plantas medicinais, alimentares e tóxicas da Ilha de Combu, Município de Belém, Estado do Pará. Rev. Bras. Farmacogn. 2005, 86: $31-30$.

4. Brasil. Ministério da Saúde (MS). Secretaria de Ciência, Tecnologia e Insumos Estratégicos. Departamento de Assistência Farmacêutica. A Fitoterapia no SUS e o Programa de Pesquisas de Plantas Medicinais da Central de Medicamentos. 2006, Brasília: MS.

5. Santos EB, Dantas GS, Santos HB, Diniz MFFM, Sampaio FC. Estudo etnobotânico de plantas medicinais para problemas bucais no município de João Pessoa, Brasil. Rev. Bras. Farmacogn. 2009, 19: 321-324.

6. Fuck SB, Athanázio JC, Lima CB, Ming LC. Plantas medicinais utilizadas na medicina popular por moradores da área urbana de Bandeirantes, PR, Brasil. Semina: Ci. Agrár. 26(3): 291-296, 2005.

7. Calábria L, Cuba GT, Hwang SM, Marra JCF, Mendonça MF, Nascimento RC, Oliveira MR, Porto JPM, Santos DF, Silva BL, Soares TF, Xavier EM, Damasceno AA, Milani JF, Rezende CHA, Barbosa AAA, Canabrava HAM. Levantamento etnobotânico e etnofarmacológico de plantas medicinais em Indianópolis, minas gerais, brasil. Rev. Bras. Plantas Med. 2008, 10(1): 49-63.

8. Arnous AH, Santos AS. Beinner RPC. Plantas medicinais de uso caseiro-conhecimento popular e interesse por cultivo comunitário. Rev. Espaço Saúde. 2005, 6(2):1-6. 
9. Rocha FAG, Araujo LSG, Lima TGD, Silva ER, Silva PA, Gundim MKM. Comércio informal de plantas medicinais: características da comercialização, crenças no uso e perfil socioeconômico dos feirantes de Lagoa Nova, RN. VII CONNEPI - Congresso Norte Nordeste de Pesquisa e Inovação, Palmas, Brasil, 2012.

10. Ming LC; Amaral Júnior, A. 2005. Aspectos etnobotânicos de plantas medicinais na reserva extrativista "Chico Mendes". The New York Botanical Garden. Disponível em <http://www.nybg.org/bsci/acre/www1/medicinal.html>. Acesso em 25 de agosto de 2015.

11. Coelho MFB et al. Medicinal plants in the neighborhood of Serra do Brigadeiro State Park, Minas Gerais State, Brazil. Acta Hortic. 2002, 569(1): 73-77.

12. Pinto EPP, Amoroso MCM, Furlan A.Conhecimento popular sobre plantas medicinais em comunidades rurais de mata atlântica-Itacaré, BA, Brasil. Acta Bot. Bras.. 2006, 20(4): 751-762.

13. Silveira SM, Cunha Júnior A, Scheuermann GN, Secchi FL, Vieira CRW. Chemical composition and antimicrobial activity of essential oils from selected herbs cultivated in the South of Brazil against food spoilage and foodborne pathogens. Ci. Rural. 2012, 47(7): 1300-1306.

14. Jesus NZT, Lima JCS, Silva RM, Espinosa MM, Martins DTO. Levantamento etnobotânico de plantas popularmente utilizadas como antiúlceras e antiinflamatórias pela comunidade de Pirizal, Nossa Senhora do Livramento-MT, Brasil. Rev. bras. farmacognia. 2009, 19(1): 130-139.

15. Politeo O, Milos MJM. Chemical composition and antioxidant capacity of free volatile aglycones from basil (Ocimum basilicum L.) compared with its essential oil. . Food Chem. 2007, 101: 379-385.

16. Telci I, Bayram E, Yilmaz G, Avci B. Variability in essential oil composition of Turkish basils (Ocimum basilicum L.). Biochem. Syst. Ecol. 2006, 34: 489-497.

17. Ozcan M, Arslan D, Unver A.Effect of drying methods on the mineral content of basil (Ocimum basilicum L.). J. Food Eng. 2005, 69: 375-379.

18. Lorenzi H, Matos FJA. Plantas Medicinais no Brasil: nativas e exóticas. Nova Odessa. 2008, São Paulo: Instituto Plantarum.

19. Monzote L, Stamberg W, Staniek K, Gille L. Toxic effects of carvacrol, caryophyllene oxide, and ascaridole from essential oil of Chenopodium ambrosioides on mitochondria. Toxicol. Appl. Pharm. 2009, 240:337-347.

20. Costa MVL, Tavares ES. Anatomia folicular de Chenopodium ambrosioides L. (Chenopodiaceae)erva-de-santa-maria. Rev. Bras. Plantas Med. 2006, 8(3): 63-71.

21. Yamashita OM, Fernandes Neto E, Campos OR, Guimarães SC. Fatores que afetam a germinação de sementes e emergência de plântulas de arruda (Ruta graveolens L.). Rev. Bras. Pl. Med. 2009, 11(2): 202-208.

22. Lorenzi H. Árvores brasileiras: manual de identificação e cultivo de plantas arbóreas do Brasil. 2. ed. Nova Odessa: Instituto Plantarum, 2002, 2: 368 p.

23. Mejri J, Abderrabba M, Mejrri M. Chemical composition of the essential oil of Ruta chalepensis L: Influence of drying, hydro-distillation duration and plant parts. Ind. Crops Prod. 2010, 32: 671-673.

24. BRASIL. Ministério da Saúde. RENISUS - Relação Nacional de Plantas Medicinais de Interesse ao SUS. Espécies vegetais. DAF/SCTIE/MS - RENISUS - fev/2009. Disponível em: <http://portal.saude.gov.br/portal/arquivos/pdf/RENISUS.pdf>. Acesso em abril de 2015

25. Pedroso K, Watzlawick LF, Oliveira NK, Valerio AF.,Gomes GS, Silvestre R. Levantamento de plantas medicinais arbóreas e ocorrência em Floresta Ombrófila Mista. Ambiciência. 2007, 3(1): 3950 .

26. Santos FCC, Monteiro SG., Vogel FS. Extrato aquoso de alho (Allium sativum) sobre nematóides gastrintestinais de ovinos Rev. Bras. Agroecol. 2012, 7(1). ISSN 1980-9735. Disponível em: <http://www.abaagroecologia.org.br/revistas/index.php/rbagroecologia/article/view/10365>. Acesso em 14 Outubro de 2015.

27. Brito, MVH, Brazão, RV, Siqueira, RBP, dos Santos, MT. Efeito do óleo de andiroba em cultura de Staphylococcus aureus e Escherichia coli: estudo in vitro. Rev. para. med. 2001,15 (1):36-40.

28. Santos LC, Amorim MMR. Uso da Aroeira (Schinus terebinthifolius Raddi) para tratamento de infecções vaginais. Femina 2002, 30:339-42.

29. Lima EO, Farias NMP, Souza E. Propriedades antibacterianas de óleos essenciais de plantas medicinais.Rev. bras. ciênc. Saúde. 2003 7(3):251-258.

30. Sakai M. Current status of fish immunostimulants. Aquaculture. 1999, 172: 63-92.

31. KobayashI H, Matsunaga K, Fuji M. PSK as a chemopreventive agent. Cancer epidem biomar. 1993, 2: 271-276.

32. Souza CD, Felfili JM. Uso de plantas medicinais na região de Alto Paraíso de Goiás, GO, Brasil. Acta Bot. Bras. 2006, 20(1): 135-142.

33. Mengue SS, Mentz LA, Schenkel EP. Uso de plantas medicinais na gravidez. Rev Bras Farmacogn. 2001, 11:21- 35 
34. Tôrres AR, Oliveira RAG, Diniz MFFM, Araújo EC. Estudo sobre o uso de plantas medicinais em crianças hospitalizadas da cidade de João Pessoa: riscos e benefícios. Rev Bras Farmacogn. 2005, 15: 373-380.

35. Teixeira SA, José IMM. "Plantas medicinais utilizadas no município de Jupi, Pernambuco, Brasil." Iheringia Série Botânica. 2014, 61: 1- 2.

36. LorenzI, H. Plantas daninhas do Brasil. Nova Odessa. 2000. Instituto Plantarum de Estudos da Flora, 3. ed.

37. Duarte MCT. Atividade antimicrobiana de plantas medicinais e aromáticas utilizadas no Brasil. MultCiência. UNICAMP-Campinas. 2006, p. 16.

38. Silva, MA, Barbosa JS. "Levantamento das plantas espontâneas e suas potencialidades fitoterapêuticas: um estudo no complexo Aluízio Campos-Campina Grande-PB." Revista Brasileira de Informações Científicas. 2010, 1: 52-66.

39. dos Santos S,Alburqueque HN, Cavalcanti MLF. "Estudo do uso de plantas medicinais pela comunidade quilombola Senhor do Bonfim-Areia-PB." Rev. Biol. Ciênc. Terra. 2009, 1: 31-36.

40. Lima EO. "Propriedades antibacterianas de óleos essenciais de plantas medicinais." Rev. bras. ciênc. Saúde. 2003, 7(3): 251-258.

41. Madaleno IM. "Plantas da medicina popular de São Luís, Brasil. Cienc. Hum. 2011, 6(2): 273-286.

42. Bandeira LRGS, da Silva MDS, Brito RCT. "Uso de plantas medicinais cultivadas na comunidade Lagoa do Porão, Jatobá do Piauí." Revista Interdisciplinar . 2015, 8(1): 55-61.

43. Santos FM dos. Aspectos ecofisiológicos de Aloysia gratissima (Gillies et Hook) Troncoso [Verbenaceae] associados à composição do óleo essencial e sua ação antimicrobiana. Dissertação (Mestrado em Fisiologia)-Universidade Federal de Lavras, Lavras, 2007. 116

44. PaulettI PM, Araujo AR, Bolzani VS, Young MC. Marx.Triterpenos de Styrax camporum (styracaceae). Quím. Nova [online]. 2002, 25(3): 349-352.

45. Lavabre, M. aromaterapia: a cura pelos óleos essenciais. $5^{\circ}$ edição, Rio de Janeiro. Record, editora Nova era. 2001, 18:89.

46. Costa, V. P.,Mayworm, MAS. Plantas medicinais utilizadas pela comunidade do bairro dos Tenentesmunicípio de Extrema, MG, Brasil. Rev. Bras. Pl. Med. Botucatu. 2011, 13(3): 282-292.

47. Leite I. A, de Morais AM, Carneiro RG, Leite, CA. A etnobotânica de plantas medicinais no município de São josé de Espinharas, Paraiba, Brasil. Biodiversidade. 2014, 14(1).

48. Da silva RM, Faria MT.. Caracterização etnobotânica e histoquímica de plantas medicinais utilizadas pelos moradores do bairro Carrilho, Goianésia (GO). Enciclopédia Biosfera. 2014, 10(19):2807.

49. Araújo IFS, Souza LFS, Guarçoni EAE, Firmo WCA. Plantas medicinais comercializadas em Bacacal, Maranhão. Natureza on line. 2015, 13 (3): 111-116.

50. Li-chen W, Nien-chu F, Ming-huI L, Inn-ray C, Shu-jung H, Chingyuan H, Shang-yu H. Antiinflammatory Effect of Spilanthol from Spilanthes acmella on Murine Macrophage by DownRegulating LPS-Induced Inflammatory Mediators. Journal Agric. Food Chemitry. 2008, 56:2341-2349.

51. Lima VB. Estudo fitoquímico de Himatathus obovatus (Muell. Arg.) Woodson (Apocynaceae): Isolamento, elucidação estrutural e atividade biológica. 2005. 174f. Tese (Doutorado em Química Orgânica) - Universidade Estadual de Campinas - Unicamp, Campinas, SP, 2005.

52. de Souza CD, Felfili JM. Uso de plantas medicinais na região de Alto Paraíso de Goiás, GO, Brasil. Acta Bot. Bras. 2006, 20(1): 135-142.

53. Chiang L. Antiviral activities of extracts and selected pure constituents of Ocimum basilicum. Clin Exp Pharmacol Physiol. 2005, 32: 811-816.

54. Freire JM. Óleos essenciais de canela, manjerona e anis-estrelado: caracterização química e atividade biológica sobre Staphylococcus aureus, Escherichia coli, Aspergillus flavus e Aspergillus parasiticus. 2008. ix, 68 p. Dissertação (Mestrado em Agroquímica) - Universidade Federal de Lavras.

55. Lemos ICS, de Andrade AM, de Menezes IRA, Fernandes GP. Uso de plantas medicinais para o tratamento de feridas. Revista Interdisciplinar. 2005 8(2): 60-67.

56. SIngh M, Rao RSG. Influence of sources and doses of $\mathrm{N}$ and $\mathrm{K}$ on herbage, oil yield and nutrient uptake of patchouli [Pogostemon cablin (Blanco) Benth.] in semi-arid tropics. Industrial Crops and Products. 2009, 29: 229-234.

57. Sousa IMO, Rodrigues RAF, Navarro Filho CV, Magalhães PM, Pereira B, Fogliuo.. Avaliação da estabilidade de extrato etanólico bruto liofilizado de Arrabidaea chica Verlot. In: Reunião anual da sociedade brasileira de química, 30., 2007. Anais.... Sociedade Brasileira de Química: SBQ, 2007. 
Disponível em <http://sec.sbq.org.br/cdrom/30ra/resumos/T0271-1.pdf> Acesso em 14 Outubro de 2015.

58. Borges KB, Bautista BH, Guilera S.). Diabetes-utilização de plantas medicinais como forma opcional de tratamento. Revista Eletrônica de Farmácia. 2008 , 5(2):12-20.

59. Langley P. Why a pomegranate? British of Medicine Journal. 2000, 321(4):1153-4.

60. Diniz VW, Dantas Filho HA, Müller RF, Fernandes KG, Palheta DC. Classificação multivariada de ervas medicinais da região amazônica e suas infusões de Acordo com sua composição mineral. Quim. Nova. 2013, 36(2): 257-261. 